\title{
CORRELATIVE BEHAVIOUR OF ANISOTROPIC BINARY LIQUID SYSTEM IN THREE-MOMENT APPROXIMATION
}

\author{
A. V. Chalyi ${ }^{1}$, A. N. Vasil'ev ${ }^{2}$ \\ ${ }^{1}$ National Medical University, Department of Physics, \\ 13 Shevchenko Blvd., Kyiv, UA-01601, Ukraine \\ ${ }^{2}$ Taras Shevchenko National University of Kyiv, Department of Theoretical Physics, \\ 6 Glushkov Pr., Kyiv, UA-03022, Ukraine
}

(Received February 25, 2002; received in final form January 21, 2003)

\begin{abstract}
To investigate the correlative properties of anisotropic binary mixture the Ornstein-Zernike system of integral equations is used. The influence of the anisotropy on the critical behaviour of the binary liquid mixture is considered by means of the first non-zero spatial moment for the direct correlation functions. The pair correlation functions in three-moment approximation are found. Basing on the expression for the pair correlation functions it is shown that at the close vicinity of the critical point correlative behaviour of binary mixture can be described in analogue with onecomponent liquids only by one correlation function. A dependence of the critical temperature on the concentration of the mixture components is considered. It is found that the anisotropy causes the shift of the critical temperature. Close to the critical point the dependence of such a shift on the effective field variable is of a scaling-law form.
\end{abstract}

Key words: correlation functions, phase transitions, critical phenomena.

PACS number(s): 05.70.Fh, 05.70.Jk

\section{INTRODUCTION}

Critical phenomena and phase transitions in binary liquid systems are of great theoretical and experimental interest [1-7]. As it is well-known at the critical state all the systems are characterized with strong susceptibility on the presence of impurities, external fields, temperature gradients and other factors. From this point of view the additional component in binary mixture in comparison with pure liquid is the point that causes some interesting effects. On the other hand, the universal behaviour of different systems at the close vicinity of the critical state allows to use the methods of scaling theory and hypothesis of isomorphism significantly simplifying analysis of those systems.

Most of the results in theory of liquids can be obtained basing on the expressions for the pair correlation functions and thus calculating them is the important task of condensed matter physics [8]. To investigate correlative behaviour of binary mixture we propose to use the Ornstein-Zernike (OZ) system of integral equations. This allows to find the asymptotic expressions for the pair correlation functions of liquid binary system. For these purposes it is necessary to transform the OZ system of integral equations to the system of differential equations.

\section{THE PAIR CORRELATION FUNCTIONS}

Let us consider the OZ system of integral equations in such a form:

$$
\begin{aligned}
g_{i j}\left(\mathbf{r}_{1}, \mathbf{r}_{2}\right) & =f_{i j}\left(\mathbf{r}_{1}, \mathbf{r}_{2}\right) \\
& +\sum_{k=1}^{2} \int \rho\left(\mathbf{r}_{3}\right) f_{i k}\left(\mathbf{r}_{1}, \mathbf{r}_{3}\right) g_{k j}\left(\mathbf{r}_{3}, \mathbf{r}_{2}\right) d \mathbf{r}_{3}
\end{aligned}
$$

where $g_{i j}\left(\mathbf{r}_{1}, \mathbf{r}_{2}\right)$ and $f_{i j}\left(\mathbf{r}_{1}, \mathbf{r}_{2}\right)$ are the pair and the direct correlation functions respectively and $\rho(\mathbf{r})$ is the density.

For the spatially homogeneous system the correlation functions depend on the distance between the points of correlation only, i.e., $f_{i j}\left(\mathbf{r}_{1}, \mathbf{r}_{2}\right)=f_{i j}\left(\mathbf{r}_{2}-\mathbf{r}_{1}\right)=$ $f_{j i}\left(\mathbf{r}_{1}-\mathbf{r}_{2}\right), g_{i j}\left(\mathbf{r}_{1}, \mathbf{r}_{2}\right)=g_{i j}\left(\mathbf{r}_{2}-\mathbf{r}_{1}\right)=g_{j i}\left(\mathbf{r}_{1}-\mathbf{r}_{2}\right)$ and $\rho(\mathbf{r})=\langle\rho\rangle=$ const. Equation (1) in this case gives:

$$
g_{i j}(\mathbf{r})=f_{i j}(\mathbf{r})+\langle\rho\rangle \sum_{k=1}^{2} \int f_{i k}\left(\mathbf{r}_{1}\right) g_{k j}\left(\mathbf{r}-\mathbf{r}_{1}\right) d \mathbf{r}_{1}
$$

Moreover, for the isotropic systems the correlation functions do not depend on the direction. This allows when deriving the differential equation to use the two even spatial moments for the direct correlation functions only [9]. In anisotropic systems (such anisotropy can be caused by the external field, for example) all the correlation functions depend on two arguments. We will consider the weak anisotropy that does not effect the homogeneity of the system. In this case the correlation functions depend on the distance between the correlating points as well as on the direction. So when deriving the differential equations we should take into account the odd moments (namely the first moment) of the direct correlation functions [10].

To find the asymptotic solution of equation (2) we 
should transform it to the system of differential equations. Keeping in mind that the direct correlation functions differs from zero only when the distance between the correlating points is small enough [9] we obtain:

$$
\begin{aligned}
& g_{i j}(\mathbf{r})=f_{i j}(\mathbf{r})+\sum_{k=1}^{2} A_{i k} g_{k j}(\mathbf{r}) \\
& -\sum_{k=1}^{2} \sum_{\alpha=1}^{3} C_{i k}^{\alpha} \frac{\partial g_{k j}(\mathbf{r})}{\partial x_{\alpha}}+\sum_{k=1}^{2} \sum_{\alpha=1}^{3} \sum_{\beta=1}^{3} B_{i k}^{\alpha \beta} \frac{\partial^{2} g_{k j}(\mathbf{r})}{\partial x_{\alpha} \partial x_{\beta}}
\end{aligned}
$$

where $A_{i j}=\langle\rho\rangle \int f_{i j}(\mathbf{r}) d \mathbf{r}, C_{i j}^{\alpha}=\langle\rho\rangle \int f_{i j}(\mathbf{r}) x_{\alpha} d \mathbf{r}$ and $B_{i j}^{\alpha \beta}=\langle\rho\rangle / 2 \int f_{i j}(\mathbf{r}) x_{\alpha} x_{\beta} d \mathbf{r}$. These matrices should commute with each other to satisfy the symmetry of the initial OZ system of integral equations.

Let us consider the correlation functions in such a form: $\hat{g}(\mathbf{r})=\hat{G}(r) \cdot \prod_{\alpha=1}^{3} \hat{L}\left(\hat{\gamma}^{\alpha}, x_{\alpha}\right)$ and $\hat{f}(\mathbf{r})=\hat{F}(r)$. $\prod_{\alpha=1}^{3} \hat{L}\left(\hat{\gamma}^{\alpha}, x_{\alpha}\right)$, where matrices $\hat{g}(\mathbf{r})=\left(g_{i j}(\mathbf{r})\right), \hat{f}(\mathbf{r})=$ $\left(f_{i j}(\mathbf{r})\right), \hat{G}(r)=\left(G_{i j}(r)\right), \hat{F}(r)=\left(F_{i j}(r)\right)$ and matrix $\hat{L}(\hat{D}, y)$ is determined with the equation:

$$
\frac{\partial \hat{L}(\hat{D}, y)}{\partial y}=\hat{D} \hat{L}(\hat{D}, y)
$$

and can be presented either as operator $\exp (\hat{D} y)$ (in this case one takes by definition $\hat{L}(\hat{D}, y) \equiv \sum_{n=0}^{\infty} \hat{D}^{n} y^{n} / n !-$ it is easy to see that $\hat{L}$ satisfies equation (4)) or as linear combination of terms $\exp \left(\lambda_{i} y\right)$ with natural supposition that at the limiting case $\hat{L}(0, y) \equiv \hat{E}$, where $\hat{E}$ is the unit matrix and $\lambda_{1,2}$ are the eigenvalues of the matrix $\hat{D}$. We take

$$
\hat{\gamma}^{\alpha}=1 / 2 \sum_{\beta=1}^{3} \hat{U}^{\alpha \beta} \hat{C}^{\beta}
$$

where $\hat{C}^{\beta}=\left(C_{i j}^{\beta}\right)$ and matrix $\hat{U}^{\alpha \beta}$ satisfies the equation

$$
\sum_{\nu=1}^{3} \hat{U}^{\alpha \nu} \hat{B}^{\nu \beta}=\hat{E} \delta^{\alpha \beta}
$$

Here $\hat{B}^{\alpha \beta}=\left(B_{i j}^{\alpha \beta}\right)$. Then equation (3) can be presented in the following form:

$$
\sum_{\alpha=1}^{3} \sum_{\beta=1}^{3} \hat{B}^{\alpha \beta} \frac{\partial^{2} \hat{G}(r)}{\partial x_{\alpha} \partial x_{\beta}}-(\hat{E}-\hat{A}+\hat{S}) \hat{G}(r)=-\hat{F}(r)
$$

where $\hat{S}=1 / 4 \sum_{\alpha=1}^{3} \sum_{\beta=1}^{3} \hat{C}^{\alpha} \hat{U}^{\alpha \beta} \hat{C}^{\beta}$. It is possible to transform equation (7) to the more simple form if we make the substitution of coordinates in way that $z$-axis coincides with the direction of the external field (direction of the anisotropy). Then $\hat{C}^{\alpha}=(0,0, \hat{C})$ and $\hat{B}^{\alpha \beta}=0$ if $\alpha \neq \beta$. It should be pointed out that in general case $\hat{B}^{11}=\hat{B}^{22} \neq \hat{B}^{33}$. To simplify the analysis let us consider the situation when the second spatial moments of the direct correlation functions are not effected by the external field and $\hat{B}^{11}=\hat{B}^{22}=\hat{B}^{33} \equiv 3 \hat{B}$ where $\hat{B}=1 / 6\langle\rho\rangle \int \hat{f}(\vec{r}) r^{2} d \vec{r}$. From equation (7) we have:

$$
\hat{B} \Delta \hat{G}(r)-(\hat{E}-\hat{A}+\hat{S}) \hat{G}(r)=-\hat{F}(r),
$$

and after Fourier transformation we obtain then

$$
\left(\hat{E} q^{2}+\hat{B}^{-1}(\hat{E}-\hat{A}+\hat{S})\right) \hat{G}(q)=\hat{B}^{-1} \hat{F}(q)
$$

Taking the direct correlation functions being proportional to the delta-function in the zero approximation, i. e., $\hat{F}(r)=1 /\langle\rho\rangle \hat{A} \delta(r)[10]$, we can get from (9) for the matrix of the pair correlation functions:

$$
\hat{G}(q)=\left(\hat{E} q^{2}+\hat{B}^{-1}(\hat{E}-\hat{A}+\hat{S})\right)^{-1} \hat{B}^{-1} \hat{A} /\langle\rho\rangle .
$$

The matrix $\left(\hat{E} q^{2}+\hat{B}^{-1}(\hat{E}-\hat{A}+\hat{S})\right)^{-1}$ can be presented as follows:

$$
\left(\hat{E} q^{2}+\hat{B}^{-1}(\hat{E}-\hat{A}+\hat{S})\right)^{-1}=\frac{\hat{\Lambda}}{q^{2}+q_{1}^{2}}+\frac{\hat{E}-\hat{\Lambda}}{q^{2}+q_{2}^{2}}
$$

where $\hat{\Lambda}=\left(\hat{E} q_{2}^{2}-\hat{B}^{-1}(\hat{E}-\hat{A}+\hat{S})\right) /\left(q_{2}^{2}-q_{1}^{2}\right)$ and $q_{i}^{2}(i=1,2)$ are the eigenvalues of the matrix $\hat{W}=$ $\hat{B}^{-1}(\hat{E}-\hat{A}+\hat{S})$. So for the isotropic part of the pair correlation function we have:

$$
\hat{G}(q)=\frac{\hat{B}^{-1} \hat{A}}{\langle\rho\rangle} \cdot\left(\frac{\hat{\Lambda}}{q^{2}+q_{1}^{2}}+\frac{\hat{E}-\hat{\Lambda}}{q^{2}+q_{2}^{2}}\right) .
$$

Thus the matrix of the pair correlation functions is:

$$
\begin{aligned}
\hat{G}(r) & =\frac{\hat{B}^{-1} \hat{A}}{4 \pi\langle\rho\rangle r}\left[\hat{\Lambda} \exp \left(-q_{1} r\right)\right. \\
& \left.+(\hat{E}-\hat{\Lambda}) \exp \left(-q_{2} r\right)\right] .
\end{aligned}
$$

Then we obtain

$$
\begin{aligned}
\hat{g}(\vec{r}) & =\frac{\hat{B}^{-1} \hat{A} \hat{L}(\hat{\gamma}, z)}{4 \pi\langle\rho\rangle r} \\
& \times\left[\hat{\Lambda} \exp \left(-q_{1} r\right)+(\hat{E}-\hat{\Lambda}) \exp \left(-q_{2} r\right)\right],
\end{aligned}
$$

where $\hat{\gamma}=1 / 2 \hat{B}^{-1} \hat{C}$. Analysis of formulae (13) and (14) allows to conclude that there is a strong increasing of fluctuation correlation at the direction of the external field (direction of the anisotropy) in comparison with the 
isotropic system, i.e. when there is no field. It is clear that if we take $\hat{C}^{\alpha}=0$ then equations (13) and (14) give the expressions for the pair correlation functions of the isotropic binary mixture [10].

\section{CLOSE VICINITY OF THE CRITICAL POINT}

Close to the critical point the expressions for the pair correlation functions can be significantly simplified.

It is well-known that for the one-component liquids at the critical point the zero spatial moment $\Phi_{0}$ of the direct correlation function $\Phi(r)$ approaches value of 1 providing the following expression for the isothermal compressibility $\beta_{T}[11]$ :

$$
\rho k T \beta_{T}=\frac{1}{1-\langle\rho\rangle \int \Phi(r) d \vec{r}}=\frac{1}{1-\Phi_{0}} .
$$

We may take the independent termodynamical variables [1-3] in way that the matrix of the zero spatial moments of the direct correlation functions $\left.\hat{W}=\hat{B}^{-1}(\hat{E}-\hat{A}+\hat{S})\right)$ for the binary mixtures close to the critical point in respect to the independent unfixed variable (let it be temperature) with fixed others has the following asymptotic behaviour:

$$
\hat{W}=\hat{a} \tau^{2 \nu}
$$

where $\tau=\left(T_{c}-T\right) / T_{c}$ is the deviation of the temperature $T$ from its critical value $T_{c}, \nu \simeq 0.63$ is the critical index and the scaling equality $\gamma=\nu(2-\eta)$ is used with $\eta=0$ in the OZ approximation [12]. It should be pointed out that results still valid in linear approximation of $\epsilon$ expansion for critical indexes. So taking into account (16) and special fixation of termodynamical variables we conclude that at the critical point the eigenvalues $q_{i}^{2}$ depend on the temperature in such a way:

$$
q_{i}^{2}=\kappa_{i}^{2} \tau^{2 \nu}
$$

where $\kappa_{i}(i=1,2)$ are the constants that do not depend on the temperature. Then equation (13) transforms as follows:

$$
\begin{aligned}
\hat{G}(r) & =\frac{\hat{B}^{-1} \hat{A}}{4 \pi\left(\kappa_{2}^{2}-\kappa_{1}^{2}\right)\langle\rho\rangle r}\left[\left(\hat{E} \kappa_{2}^{2}-\hat{a}\right) \exp \left(-\kappa_{1} \tau^{\nu} r\right)\right. \\
& \left.-\left(\hat{E} \kappa_{1}^{2}-\hat{a}\right) \exp \left(-\kappa_{2} \tau^{\nu} r\right)\right] \\
& =\frac{\hat{B}^{-1} \hat{A} \exp \left(-\kappa_{0} \tau^{\nu} r\right)}{4 \pi\langle\rho\rangle r}[1+o(\tau)] \\
& \approx \frac{\hat{B}^{-1} \hat{A} \exp \left(-\kappa_{0} \tau^{\nu} r\right)}{4 \pi\langle\rho\rangle r}
\end{aligned}
$$

where $\kappa_{0}=\min \left(\kappa_{1}, \kappa_{2}\right)$ and correlation length $\xi=$ $\kappa_{0}^{-1} \tau^{-\nu}$ grows to infinity at the critical point. So at the close vicinity of the critical point for the binary mixture all the pair correlation functions have the same form that is similar to the OZ asymptotic expression for the pair correlation function of the one-component liquid [9]. As it is seen from (18), the correlation length is quite the same for the different correlation functions of binary mixtures. This result confirms hypothesis of isomorphism of the binary mixtures and pure liquids critical behaviour.

\section{CRITICAL TEMPERATURE}

It is naturally to consider how the critical temperature of binary mixture depends on the concentration of the components and how the anisotropy effects on it.

\section{A. Dependence of the critical temperature on concentration}

Close to the critical state the matrix $\hat{W}$ depends on the temperature in agreement with the equation (16). We may present the diagonal elements of the matrix $\hat{W}$ as follows:

$$
\begin{aligned}
& W_{11}=1-\mu x(1-t)^{2 \nu} \\
& W_{22}=1-\lambda(1-x) t^{2 \nu}
\end{aligned}
$$

where $t=\left(T-T_{2}\right) /\left(T_{1}-T_{2}\right), T_{1}$ and $T_{2}$ are the critical temperatures of the pure components, concentration $x=\left\langle\rho_{1}\right\rangle /\langle\rho\rangle, \mu$ and $\lambda$ are the constants that do not depend on the temperature and concentration. Then at the critical point we obtain the dependence of the critical temperature of the binary mixture $T_{c}(x)$ on the concentration:

$$
T_{c}(x)=\frac{x^{1 / 2 \nu} T_{1}+\chi(1-x)^{1 / 2 \nu} T_{2}}{x^{1 / 2 \nu}+\chi(1-x)^{1 / 2 \nu}},
$$

where parameter $\chi=(\lambda / \mu)^{1 / 2 \nu}$ characterizes the interaction of the components. It is clearly seen from (21) that the critical temperature $T_{c}=T_{1}$ for $x=1$ and $T_{c}=T_{2}$ for $x=0$, respectively. Dependence of the critical temperature on the concentration $x$ is shown in Fig. 1. It should be pointed out that equation (21) was obtained in supposition that the temperature dependence of the zero spatial moments of the direct correlation functions has the form of equations (19), (20). This is correct at the close vicinity of the critical points of the pure components only. So, formula (21) may be used when the temperature interval $\left|T_{1}-T_{2}\right|$ is not too large or when the concentration $x \rightarrow 0$ or $x \rightarrow 1$. In another cases, i. e., for the wide vicinity of the critical point, we should take into account next terms in the temperature dependence of the zero moments of the direct correlation functions. 


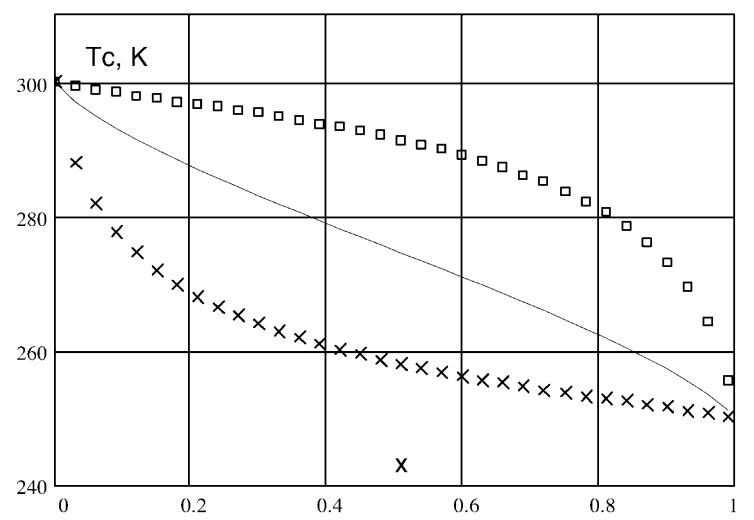

Fig. 1. Dependence of the critical temperature on the concentration. Here it is assumed $T_{1}=250 \mathrm{~K}, T_{2}=300 \mathrm{~K}$ and line - for $\chi=1$, squares - for $\chi=5$ and crosses - for $\chi=0.2$

\section{B. Influence of anisotropy on the critical temperature}

Anisotropy causes shift of the critical temperature in comparison with the isotropic mixture. To obtain the formulae for the pair correlation functions of the isotropic mixture we should take $\hat{\gamma}=0$ in equations (13) and (14). Then for the isotropic mixture we have $\hat{W} \rightarrow \hat{W}^{*}=\hat{B}^{-1}(\hat{E}-\hat{A})$. Let $T_{c}^{*}$ be the critical temperature of the isotropic mixture. We will suppose that at the close vicinity of the critical state of the isotropic system

$$
\hat{W}^{*}=\hat{a}^{*} \tau^{* 2 \nu}
$$

where $\tau^{*}=\left(T_{c}^{*}-T\right) / T_{c}^{*}$ and matrix $\hat{a}^{*}$ does not depend on the temperature. Taking into account that

$$
\hat{W}^{*}=\hat{W}+\hat{B}^{-1} \hat{S}
$$

and keeping in mind dependence (16) for the matrix $\hat{W}$ we have

$$
T_{c}=T_{c}^{*}(1-\theta(h))
$$

where function $\theta(h)=\left(\operatorname{Sp}\left(\hat{B}^{-1} \hat{S}\right) / \operatorname{Sp}\left(\hat{a}^{*}\right)\right)^{1 / 2 \nu}$ depends on the effective external field $h$ (this effective filed characterizes the anisotropy of the system). Such dependence can be estimated if we take into account that at the critical point the correlation length $\xi$ is the only characteristic size for the system. According to the results of the scaling theory [13] we may conclude that at the critical point $\operatorname{Sp}\left(\hat{B}^{-1} \hat{S}\right) / \operatorname{Sp}(\hat{a}) \sim \xi^{-2}$. At the critical isotherm we obtain for the correlation length $[11,13]$ :

$$
\xi \sim h^{-\nu / \beta \delta}
$$

where $\beta=0.33$ and $\delta=4.5$ are the critical indices. Then we have

$$
\Delta T=T_{c}^{*}-T_{c}=\theta_{0} h^{1 / \beta \delta},
$$

where $\theta_{0}$ is the constant that does not depend on $h$. Thus, anisotropy (that characterized by the effective external field $h$ ) causes the shift of the critical temperature in comparison with the isotropic mixture. It is clearly seen from (26) that if $h=0$, i.e., for the isotropic system, there is no shift of the critical temperature.

\section{CONCLUSIONS}

We have found the expressions for the pair correlation functions of the binary mixture that are valid in a wide region of the critical state. It is shown that at the close vicinity of the critical point these pair correlation functions demonstrate the universal behaviour. This result confirms the ideas of isomorphism of critical phenomena in binary mixtures and one-component liquids. It is also found that anisotropy causes the shift of the critical temperature in comparison with the isotropic system. A dependence of this shift on the effective field is of a scaling-law form.
[1] M. E. Fisher, Phys. Rev. 176, 257 (1968).

[2] R. B. Griffiths, J. C. Wheeler, Phys. Rev. A 2, 1047 (1970).

[3] M. Anisimov, Critical Phenomena in Liquids and Liquid Crystals [Kriticheskii javleniia v zhidkostyakh i zhidkikh kristalakh] (Nauka, Moscow, 1987).

[4] N. B. Wilding, Phys. Rev. E 55, 6624 (1997).

[5] N. B. Wilding, F. Shmid, P. Nielaba, Phys. Rev. E 58, 2201 (1998).

[6] Ya. Chushak, A. Baumketner, Eur. Phys. J. B 7129 (1999).

[7] F. Formisano, J. Teixeira, Eur. Phys. J. E 1, 1 (2000).
[8] C. A. Croxton, Liquid State Physics (Cambridge University Press, 1974).

[9] A. Munster, Thermodynamics of Irreversible Precesses [Termodinamika neobratimykh protsessov] (Foreing Literature Publ. House, Moscow, 1962).

[10] A. V. Chalyi et al, Condens. Matter Phys. 3, 335 (2000).

[11] H. Stenley, Introduction To Phase Transitions And Critical Phenomena (Clarendon Press, Oxford, 1971).

[12] M. E. Fisher, Critical Phenomena (Academic, New York, 1971).

[13] A. Z. Patashinskii, V. L. Pokrovskii, Fluctuation Theory of Phase Transitions (Pergamon Press, Oxford, 1979). 


\title{
КОРЕЛЯЩЙНА ПОВЕДІНКА АНІЗОТРОПНОЇ БІНАРНОЇ РІДКОЇ СИСТЕМИ В ТРИМОМЕНТНОМУ НАБЛИЖЕННІ
}

\author{
О. В. Чалий ${ }^{1}$, О. М. Васильєв ${ }^{2}$ \\ ${ }^{1}$ Начіональний медичний університет імені акад. О.О. Богомольйя, \\ кафедра медичной та біологічной фізики \\ бульв. ІІІевченка, 13, Киӥв, О1601, Украӥна \\ e-mail: avchal@iatp.kiev.ua \\ ${ }^{2}$ Кийбсъкий національний університет імені Тараса IIIеєченка, \\ фізичний факультет, кафедра теоретичной фізики \\ просп. Глушкова, 6, Киӥв, 0Зо22, Украӥна \\ e-mail:vasilal@phys.univ.kiev.ua
}

\begin{abstract}
Для дослідження корелящійних властивостей анізотропної бінарної суміші використано систему інтегральних рівнянь Орнштайна-Џерніке. Вплив анізотропії на критичну поведінку такої системи враховано через перший просторовий момент прямої корелящійної функції. Знайдено вирази для парних кореляційних функцій у тримоментному наближенні. Показано, що в околі критичного стану парні корелящії бінарної системи, за аналогією з однокомпонентними системами, можуть бути описані за допомогою лише однієі кореляпійної функпії. Розглянуто залежність критичної температури суміші від конщентрапії компонентів. Установлено, що анізотропія викликає зсув критичної температури.
\end{abstract}

